\title{
Edge States and Topological Insulating Phases Generated by Curving a Nanowire with Rashba Spin-Orbit Coupling
}

\author{
Paola Gentile, ${ }^{1}$ Mario Cuoco, ${ }^{1}$ and Carmine Ortix ${ }^{2,3}$ \\ ${ }^{1}$ CNR-SPIN and Dipartimento di Fisica "E. R. Caianiello", Università degli Studi di Salerno, \\ Via Giovanni Paolo II 132, I-84084 Fisciano (Salerno), Italy \\ ${ }^{2}$ Institute for Theoretical Solid State Physics, IFW-Dresden, Helmholtzstraße 20, D-01069 Dresden, Germany \\ ${ }^{3}$ Institute for Theoretical Physics, Center for Extreme Matter and Emergent Phenomena, Utrecht University, \\ Leuvenlaan 4, 3584 CE Utrecht, Netherlands
}

(Received 23 June 2015; published 16 December 2015)

\begin{abstract}
We prove that curvature effects in low-dimensional nanomaterials can promote the generation of topological states of matter by considering the paradigmatic example of quantum wires with Rashba spinorbit coupling, which are bent in a nanoscale periodic serpentine structure. The effect of the periodic curvature generally results in the appearance of insulating phases with a corresponding novel butterfly spectrum characterized by the formation of finite measure complex regions of forbidden energies. When the Fermi energy lies in the gaps, the system displays localized end states protected by topology. We further show that for certain superstructure periods the system possesses topologically nontrivial insulating phases at half filling. Our results suggest that the local curvature and the topology of the electronic states are inextricably intertwined in geometrically deformed nanomaterials.
\end{abstract}

DOI: 10.1103/PhysRevLett.115.256801

PACS numbers: 73.63.Nm, 03.65.Vf, 73.21.Cd, 73.43.-f

Introduction.-In recent years, topological nontrivial states of matter have been a subject of growing interest [1-3]. Topologically nontrivial electronic phases were discovered in time-reversal invariant insulators-leading to the quantum spin Hall (QSH) effect in two-dimensional systems [4-6], and to the existence of protected two-dimensional Dirac cones on the surface of three-dimensional topological insulators [7-10] —as well as in insulators with additional specific crystal point group symmetries [11]. Likewise, topological states in superconducting systems have been intensively studied since their midgap excitations can be potentially used to encode quantum bits with an unusually long coherence time $[12,13]$. Among the different solid-state platforms where topological quantum states of matter are expected to arise, low-dimensional semiconductor nanomaterials undoubtedly play a primary role. The QSH effect, for instance, was first theoretically predicted [6] and later experimentally proved in HgTe quantum wells [14]. A heterostructure comprising a semiconductor nanowire with strong spin-orbit coupling and a conventional $s$-wave superconductor has been suggested to host a topological superconducting phase $[15,16]$ with signatures of midgap Majorana bound states already reported [17].

Apart from these conventional material geometries, rapid advances in nanostructuring techniques have enabled

Published by the American Physical Society under the terms of the Creative Commons Attribution 3.0 License. Further distribution of this work must maintain attribution to the author(s) and the published article's title, journal citation, and DOI. the synthesis of novel low-dimensional nanostructures in which semiconductor nanomaterials can be bent into curved, deformable objects such as spiral-like nanotubes [18,19], nanohelices [20], and even complex nanoarchitectures resembling structures that form naturally in the most basic forms of life [21]. On one hand, these nextgeneration nanomaterials carry an enormous potential in electronics, ranging from flexible displays to the integration of semiconductor electronics with the soft, curvilinear surfaces of the human body $[22,23]$. On the other hand, the very fundamental quantum mechanical properties of the charge carriers in these nanomaterials are strongly affected by the curved background in which they live [24]. Consequently, unique curvature-induced electronic and transport properties have been identified. These include, but are not limited to, the appearance of winding-generated bound states [25], and an extremely large anisotropic magnetoresistance in nonmagnetic and spin-orbit-free semiconducting rolled-up nanotubes [26].

A relevant question that naturally arises is whether and under what circumstances the interplay between curvature effects on the electronic properties and the topology of the ground state of a low-dimensional solid-state system can be significant. In this Letter, we show not only that such interplay is relevant but also that the curved geometry of a bent nanomaterial can promote the generation of nontrivial edge states and topological insulating phases. We prove the assertion above by considering the simple example of a nanowire with Rashba spin-orbit coupling which is curved to acquire a "serpentinelike" planar periodic shape [cf. Fig. 1(a)]. The ensuing periodic 
(a) $y$

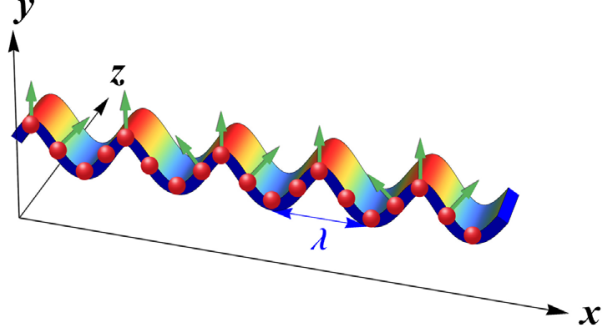

(b)

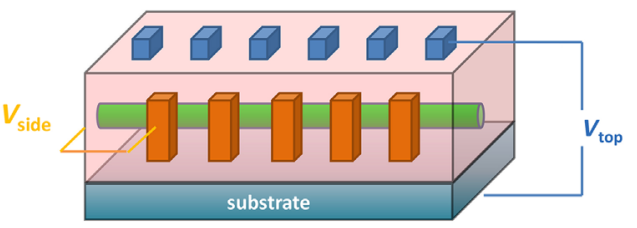

FIG. 1 (color online). (a) Schematic view of a planar serpentine nanowire with superstructure period $\lambda$. The red spheres indicate atomic sites, while the green arrows are the corresponding local directions of the Rashba spin-orbit field axis. (b) Schematic of an electrically controlled nanowire device with periodic canting of the spin-orbit axis. Top and side finger gates are used to generate an inhomogeneous electric field constant in magnitude with a varying direction in the plane perpendicular to the wire axis.

canting of the spin-orbit field axis leads to a miniband structure and a correspondent metal-insulator transition at different filling fractions. This, in turn, leads to a butterfly spectrum that, however, is neither of the Hofstadter type [27] nor of the Moth type [28]. A subsequent analysis of the system with open boundary conditions shows the occurrence of midgap edge states, which we show to be topological in nature. Finally, for certain superstructure periods, the system can display topologically nontrivial insulating phases at half filling.

Metal-insulator transition.-Our starting point is an effective continuum $\mathbf{k} \cdot \mathbf{p}$ model for a planarly curved quantum wire with a Rashba spin-orbit term originating from an electric field pointing in the direction perpendicular to the plane of the wire [the $\hat{z}$ axis in Fig. 1(a)]. In its symmetric, Hermitian form it reads

$$
\mathcal{H}_{\mathbf{k} \cdot \mathbf{p}}=-\frac{\hbar^{2}}{2 m^{\star}} \partial_{s}^{2}-\frac{i \alpha_{R}}{2}\left[\tau_{N}(s) \partial_{s}+\partial_{s} \tau_{N}(s)\right],
$$

where $s$ is the arclength of the bent nanowire measured from a reference point, $m^{\star}$ is the effective mass of the charge carriers, and $\alpha_{R}$ is the strength of the Rashba spin-orbit coupling. Finally, $\tau_{N}(s)$ is a local Pauli matrix comoving with the electrons as they propagate along $s$, and it explicitly reads $\tau_{N}(s)=\boldsymbol{\tau} \cdot \hat{\mathcal{N}}(s)$, where $\hat{\mathcal{N}}(s)$ is the local normal direction of the curved wire, while the $\tau$ 's are the usual Pauli matrices. The Hamiltonian in Eq. (1) corresponds to the effective continuum model for the curved quantum wires of Refs. $[29,30]$ in the absence of strain-induced effects, and it can be mapped to the model for a conventional quantum wire with a locally varying spin-orbit field axis (cf. Fig. 1). The latter can be determined by writing the normal direction in the Euclidean space in terms of an angle $\theta(s)$ as $\hat{\mathcal{N}}(s)=$ $\{\sin \theta(s), \cos \theta(s), 0\}$, and using the Frenet-Serret-type equation of motion $\partial_{s} \hat{\mathcal{N}}(s)=-\kappa(s) \hat{\mathcal{T}}(s)$, with $\hat{\mathcal{T}}(s)=$ $\{\cos \theta(s),-\sin \theta(s), 0\}$ being the tangential direction and $\kappa(s)$ the local curvature. It then follows that the local direction of the spin-orbit field axis is entirely determined by the curvature of the quantum wire via $\theta(s)=$ $-\int s \kappa\left(s^{\prime}\right) d s^{\prime}$. For a periodic serpentine quantum wire, it also implies that the spin-orbit field axis undergoes a periodic canting with the maximum canting angle proportional to the curvature. We emphasize that, because of the gate tunability of the Rashba coupling, a periodic canting of the spin-orbit field axis can be equally realized in "conventional" (straight) quantum wires with local top and side finger gates [cf. Fig. 1(b)] that create an overall electric field constant in strength but whose direction undergoes periodic tilts in the plane perpendicular to the wire axis. Albeit requiring a number of nontrivial design criteria, this device setup does not go beyond present-day experimental capabilities.

In order to study the effect of a periodic canting of the spin-orbit field axis on the electronic properties of a quantum wire, we next introduce a tight-binding model obtained by discretizing Eq. (1) on a lattice. It can be written as

$$
\mathcal{H}=\sum_{j} \sum_{\sigma, \sigma^{\prime}=\uparrow, \downarrow} c_{j, \sigma}^{\dagger}\left(t \delta_{\sigma, \sigma^{\prime}}+\hat{\alpha}_{j, j+1}^{\sigma, \sigma^{\prime}}\right) c_{j+1, \sigma^{\prime}}+\text { H.c. },
$$

where $c_{j, \sigma}^{\dagger}, c_{j, \sigma}$ are operators creating and annihilating, respectively, an electron at the $j$ th site with spin projection $\sigma=\uparrow, \downarrow$ along the $z$ axis, $t$ is the hopping amplitude between nearest-neighbor sites, and the spin-dependent nearest-neighbor hopping amplitudes are

$$
\hat{\alpha}_{j, j+1}=i \alpha_{R}\left[\tau_{x} g_{j}^{x}+\tau_{y} g_{j}^{y}\right] .
$$

In the equation above, $g_{j}^{x}=\sin \theta\left(s_{j}\right)+\sin \theta\left(s_{j+1}\right)$ and $g_{j}^{y}=\cos \theta\left(s_{j}\right)+\cos \theta\left(s_{j+1}\right)$, which are determined by the position of the atoms along the quantum wire and the specific geometrical shape. For the latter, we assume a simple sinusoidal form with parametric equation in Euclidean space $\mathbf{r}=\{x, A \sin (2 \pi x) / \lambda, 0\}$, with period $\lambda$ and height $A$. The atomic positions can be instead written as $s_{j} / \lambda=p j / q+\varphi /(2 \pi)$, where $p$ and $q$ are integers whose ratio is $p / q=a / \lambda$ (with $a$ being the lattice constant), while $\phi \in[0,1]$ accounts for nonequivalent displacements of the atoms in one superstructure period. As a result, the local spin-dependent hopping amplitudes render a one-dimensional superlattice with superlattice constant $p \lambda$. Although $p / q \ll 1$ in a serpentine quantum wire with superstructure periods at the tens of nanometers scale, we will extend our analysis to the full range $0<p / q<1$ since, as mentioned 


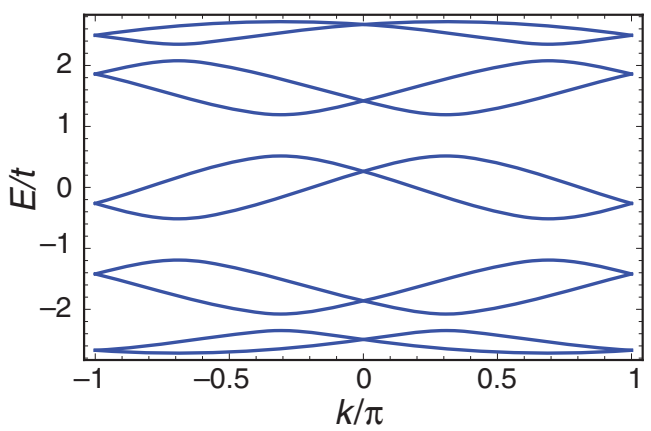

FIG. 2 (color online). Band structure of a nanocorrugated wire with Rashba spin-orbit coupling in the first $\mathrm{mBZ}$ for $p / q=1 / 5$, $\alpha_{R}=0.6 t, A / \lambda=1$, and the displacement field $\phi=0$. Momenta are measured in units of $1 / \lambda$. The canting of the spin-orbit field axis leads to band gap openings at unpinned momenta in the $\mathrm{mBZ}$.

above, Eq. (2) applies to quantum wires with opportunely designed finger gates.

Figure 2 shows the band structure for $p / q=1 / 5$ in the first mini-Brillouin zone (mBZ) $k \in[-\pi /(p \lambda), \pi /(p \lambda)]$. The presence of the spin-orbit interaction terms removes the spin degeneracy of the minibands except at the timereversal $\mathcal{T}$ invariant points $k=0, \pi /(p \lambda)$, where the eigenstates are Kramers degenerate. This, however, does not preclude $a$ priori band gaps opening at different points in the mBZ. And, indeed, Fig. 2 shows the occurrence of full band gaps at the filling fractions $\nu=n / q$, with an $n$ integer, whose magnitude monotonically increases with the corrugation height $A$. We find the opening of band gaps for finite values of the corrugation height $A$ to occur for $p / q=$ $1 / 6,1 / 7$ (see the Supplemental Material [31]). Henceforth, the periodic buckling of the nanowire generally induces a metal-insulator transition, and thus defines a nanoflex transistor switch, the status of which is "on" when the nanowire is flat and "off" when the nanowire is planarly curved. A similar nanoscale transistor switch has been suggested in nanohelices without spin-orbit coupling but subject to an externally applied rotating electric field [32].

Butterfly spectrum and topological edge states.-To further verify that the metal-insulator transition is a generic feature due to the periodic canting of the Rashba spin-orbit field axis, we have diagonalized the Hamiltonian in Eq. (2) for different commensurate superlattices, changing the displacement field $\phi$ in the $[0,1]$ interval while keeping the ratio $A / \lambda$ fixed. Figure 3 shows the ensuing energy spectrum as a function of the ratio between the atomic lattice constant and the superlattice period. It exhibits two main holes of forbidden energies with a double wing shape joining at $p / q=1 / 4$ and $p / q=3 / 4$. The generation of replicas of such twin wings of holes of finite measure and various sizes, the largest of which join at $p / q=(2 n+1) / 8$, evidences a self-similar "butterflylike" structure of the electronic spectrum. Although a rigorous proof cannot be made, this also reminds one of a fractal structure. Furthermore, the present

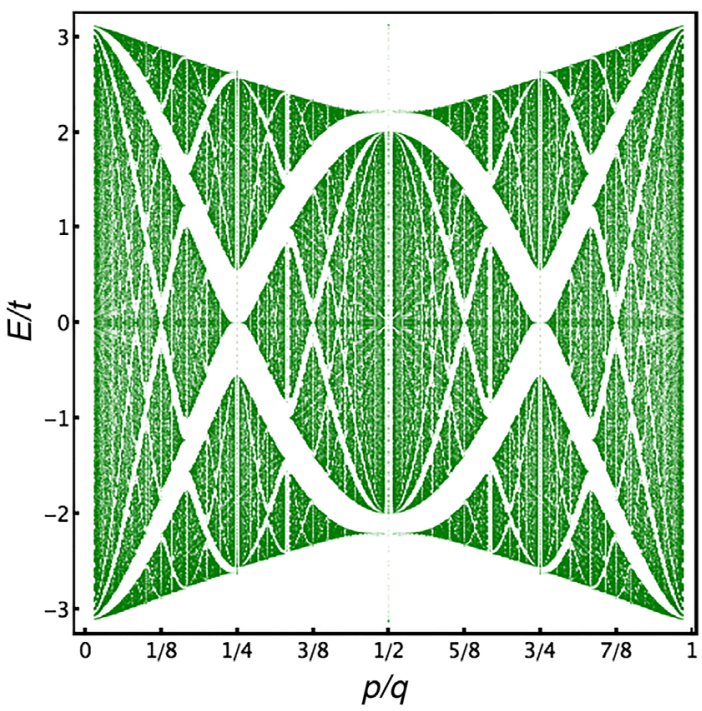

FIG. 3 (color online). Butterfly spectrum for the superlattice system of Eq. (2) as a function of $p / q$. We have set the corrugation height at $A=5 \lambda$. The spectrum clearly exhibits a self-similar structure.

spectrum is manifestly different in shape from both the Hofstadter butterfly realized in conventional two-dimensional electron gases [27], graphene moiré superlattices [33], and one-dimensional optical superlattices [34,35] and the moth butterfly discussed in the context of cold atoms in nonAbelian gauge potentials [28].

Having established the ubiquitous presence of insulating phases in our serpentine nanowire, we have then analyzed the possible occurrence of localized edge states within the curvature-induced gaps while sweeping the displacement field $\phi$, as is routinely done in one-dimensional superlattices with an additional (dynamical) parameter

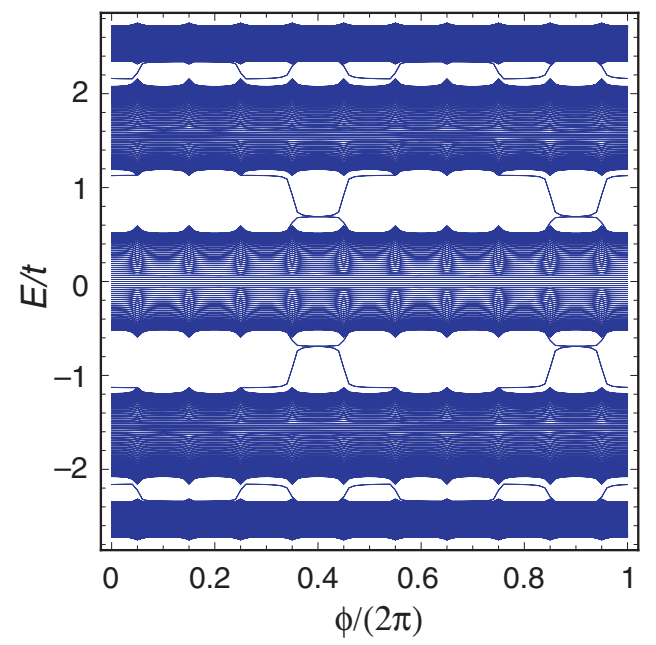

FIG. 4 (color online). Energy spectrum of the model Hamiltonian in Eq. (2) for $p / q=1 / 5, \quad \alpha_{R}=0.6$, and $A / \lambda=1$. The spectrum has been obtained by exact diagonalization of the Hamiltonian for a finite atomic chain containing $N=650$ atoms and open boundary conditions. 


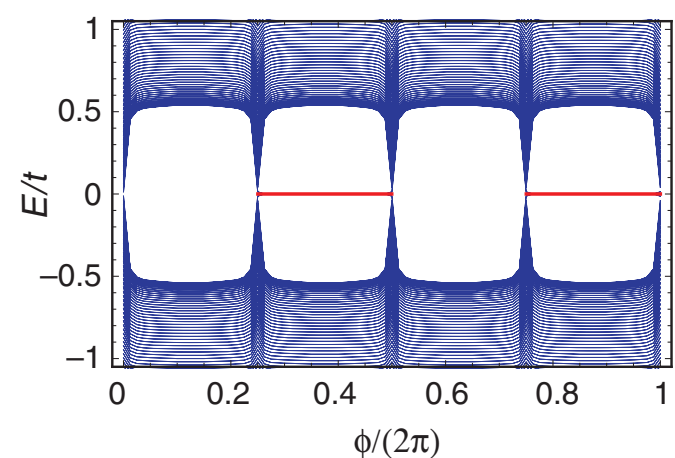

FIG. 5 (color online). Energy spectrum of the model Hamiltonian in Eq. (2) for $p / q=1 / 4, \alpha_{R}=0.6$, and $A / \lambda=1$. The spectrum has been obtained by exact diagonalization of the Hamiltonian for a finite atomic chain containing $N=640$ atoms and open boundary conditions.

[36-40]. Figure 4 shows the energy spectrum for a finite size system with open boundary conditions considering the superlattice periods $\lambda=5 a$. Within all gaps, there appear edge states localized at the left and right boundaries of the atomic chain whose energy changes continuously with $\phi$, connecting the valence to the conduction bulk bands. Since the appearance of such edge states can generally be attributed to nontrivial topological properties of bulk systems, we have then analyzed the topological character of the ground state of the system, with the displacement field $\phi$ playing the role of an artificial dimension [41]. It can be shown that the addition of this extra dimension breaks the "time-reversal" symmetry constraint $\Theta^{-1} \mathcal{H}(k, \phi) \Theta \neq \mathcal{H}(-k,-\phi)$, thereby allowing for insulating phases with nonzero Chern numbers. We have therefore computed the Chern numbers in the insulating phases of Fig. 2 using the method outlined in Ref. [42] and have found Chern number $C= \pm 4$ and $C= \pm 8$ for the two central and two exterior gaps, respectively. This result is in perfect agreement with the foregoing analysis of the spectrum with open boundary conditions, which, depending on the filling fraction, indeed shows four or eight edge states per edge composed of the two spin species (cf. Fig. 4). We have verified that in-gap edge states protected by a nonzero Chern number also appear at different values of $p / q$ (see the Supplemental Material [31]), thereby directly proving the general occurrence of edge states topological in nature in a serpentine quantum wire.

Topological insulating phases. - Finally, we show that a periodic canting of the Rasha spin-orbit field axis can also lead to one-dimensional topological insulating phases. This occurs at the central points of the wings in our butterfly spectrum of Fig. 3. For all values of $\phi$, the system possesses time-reversal symmetry $\Theta^{-1} \mathcal{H}(k, \phi) \Theta=\mathcal{H}(-k, \phi)$, with $\Theta^{2}=-1$ as required for spin-one-half fermions, a unitary chiral symmetry $\mathcal{C}^{-1} \mathcal{H}(k, \phi) \mathcal{C}=-\mathcal{H}(k, \phi)$, and an antiunitary symplectic particle-hole symmetry $\mathcal{P}^{-1} \mathcal{H}(k, \phi) \mathcal{P}=$ $-\mathcal{H}(-k, \phi)$, with $\mathcal{P}^{2}=-1$. This also implies that at these selected $p / q$ values, the system is in the chiral symplectic symmetry class CII of the Altland-Zirnbauer classification $[43,44]$, and it thus possesses an integer $\mathcal{Z}$ topological invariant, provided it is insulating at half filling. In terms of the displacement field $\phi$, we find that the half-filling gap has an even $q$ number of closing-reopening points (cf. Fig. 5 and the Supplemental Material [31]), which is an unambiguous signal of the occurrence of a topological phase transition between insulating phases with different $\mathcal{Z}$ invariant. To verify this point, we have thus considered a superlattice system with $\lambda=4 a$ and open boundary conditions. The ensuing energy spectrum as a function of the displacement field $\phi$ is shown in Fig. 5. It shows the presence of two spin-degenerate topologically protected zero-energy modes between the gap closing-reopening points, thereby explicitly proving that the geometric displacement of the atoms in a nanocorrugated nanowire drives a topological phase transition between a $\mathcal{Z}=2$ and a $\mathcal{Z}=0$ insulating state. Precisely the same feature is encountered at the central points of the wing replicas in the butterfly spectrum of Fig. 3 (see the Supplemental Material [31]).

Conclusions.-To sum up, we have analyzed curvature effects on the electronic states of a serpentine quantum wire with Rashba spin-orbit interaction. We have shown that the ensuing periodic canting of the Rashba spin-orbit field yields a novel butterfly spectrum with insulating phases characterized by the presence of edge states, which are topological in origin. We have also shown that the curved geometry of such a quantum wire can promote the onset of one-dimensional topological insulating phases with topologically protected zero end modes. There are numerous nanostructuring methods to experimentally fabricate nanowires with complex geometrical shapes, including electron beam lithography [45], adhesion of ribbons on prestrained substrates [46], and electrodeposition onto self-organized carbon nanotubes [47], suggesting that our findings can be experimentally tested in present-day nanodevice setups. Using realistic parameters for an InAs (lattice spacing $a \simeq 6 \AA$ A) nanowire, $t \simeq 1 \mathrm{eV}$ [48], $\alpha_{R} \simeq 50 \mathrm{meV}$ [49], we obtain for a serpetine nanowire with $A=\lambda \simeq 40 \mathrm{~nm}$ a bulk gap $\simeq 0.2 \mathrm{meV}$. Thus, the upper bound for detecting topological edge states is for temperatures in the achievable range of a few kelvin. Our results constitute a proof-ofprinciple demonstration that the geometric curvature of bendable nanomaterials can impact the topology of the electronic states in low-dimensional systems, and it can thus inaugurate the search for other topological nontrivial states of matter in geometrically deformed nanostructures.

We acknowledge the financial support of the Future and Emerging Technologies (FET) program within the Seventh Framework Programme for Research of the European Commission, under FET-Open Grant No. 618083 (the CNTQC project). C. O. thanks the Deutsche Forschungsgemeinschaft (Grant No. OR 404/1-1) for support. 
[1] M. Z. Hasan and C. L. Kane, Rev. Mod. Phys. 82, 3045 (2010).

[2] J. E. Moore, Nature (London) 464, 194 (2010).

[3] X.-L. Qi and S.-C. Zhang, Rev. Mod. Phys. 83, 1057 (2011).

[4] C. L. Kane and E. J. Mele, Phys. Rev. Lett. 95, 146802 (2005).

[5] C. L. Kane and E. J. Mele, Phys. Rev. Lett. 95, 226801 (2005).

[6] B. A. Bernevig, T. L. Hughes, and S.-C. Zhang, Science 314, 1757 (2006).

[7] L. Fu, C. L. Kane, and E. J. Mele, Phys. Rev. Lett. 98, 106803 (2007).

[8] D. Hsieh, D. Qian, L. Wray, Y. Xia, Y. S. Hor, R. J. Cava, and M.Z. Hasan, Nature (London) 452, 970 (2008).

[9] H. Zhang, C.-X. Liu, X.-L. Qi, X. Dai, Z. Fang, and S.-C. Zhang, Nat. Phys. 5, 438 (2009).

[10] Y. L. Chen, J. G. Analytis, J.-H. Chu, Z. K. Liu, S.-K. Mo, X. L. Qi, H. J. Zhang, D. H. Lu, X. Dai, Z. Fang, S. C. Zhang, I. R. Fisher, Z. Hussain, and Z.-X. Shen, Science 325, 178 (2009).

[11] L. Fu, Phys. Rev. Lett. 106, 106802 (2011).

[12] J. Alicea, Rep. Prog. Phys. 75, 076501 (2012).

[13] C. W. J. Beenakker, Annu. Rev. Condens. Matter Phys. 4, 113 (2013).

[14] M. König, S. Wiedmann, C. Brüne, A. Roth, H. Buhmann, L. W. Molenkamp, X.-L. Qi, and S.-C. Zhang, Science 318, 766 (2007).

[15] R. M. Lutchyn, J. D. Sau, and S. Das Sarma, Phys. Rev. Lett. 105, 077001 (2010).

[16] Y. Oreg, G. Refael, and F. von Oppen, Phys. Rev. Lett. 105, 177002 (2010).

[17] V. Mourik, K. Zuo, S. M. Frolov, S. R. Plissard, E. P. A. M. Bakkers, and L.P. Kouwenhoven, Science 336, 1003 (2012).

[18] V. Y. Prinz, V. A. Seleznev, A. K. Gutakovsky, A. V. Chehovskiy, V. V. Preobrazhenskii, M. A. Putyato, and T. A. Gavrilova, Physica (Amsterdam) 6E, 828 (2000).

[19] O. G. Schmidt and K. Eberl, Nature (London) 410, 168 (2001).

[20] H.-F. Zhang, C.-M. Wang, and L.-S. Wang, Nano Lett. 2, 941 (2002).

[21] S. Xu et al., Science 347, 154 (2015).

[22] J. A. Rogers, M. G. Lagally, and R. G. Nuzzo, Nature (London) 477, 45 (2011).

[23] J.-W. Jo, J. Kim, K.-T. Kim, J.-G. Kang, M.-G. Kim, K.-H. Kim, H. Ko, Y.-H. Kim, and S. K. Park, Adv. Mater. 27, 1182 (2015).

[24] B. S. DeWitt, Rev. Mod. Phys. 29, 377 (1957).

[25] C. Ortix and J. van den Brink, Phys. Rev. B 81, 165419 (2010).
[26] C.-H. Chang, J. van den Brink, and C. Ortix, Phys. Rev. Lett. 113, 227205 (2014).

[27] D. R. Hofstadter, Phys. Rev. B 14, 2239 (1976).

[28] K. Osterloh, M. Baig, L. Santos, P. Zoller, and M. Lewenstein, Phys. Rev. Lett. 95, 010403 (2005).

[29] P. Gentile, M. Cuoco, and C. Ortix, SPIN 03, 1340002 (2013).

[30] C. Ortix, Phys. Rev. B 91, 245412 (2015).

[31] See Supplemental Material at http://link.aps.org/ supplemental/10.1103/PhysRevLett.115.256801 for the electronic bandstructure of a nanocorrugated quantum wire in the presence of Rashba spin-orbit interaction with other representative values of the corrugation period.

[32] X.-L. Qi and S.-C. Zhang, Phys. Rev. B 79, 235442 (2009).

[33] L. A. Ponomarenko, R. V. Gorbachev, G. L. Yu, D. C. Elias, R. Jalil, A. A. Patel, A. Mishchenko, A. S. Mayorov, C. R. Woods, J. R. Wallbank, M. Mucha-Kruczynski, B. A. Piot, M. Potemski, I. V. Grigorieva, K. S. Novoselov, F. Guinea, V. I. Fal'Ko, and A. K. Geim, Nature (London) 497, 594 (2013).

[34] M. Aidelsburger, M. Atala, M. Lohse, J. T. Barreiro, B. Paredes, and I. Bloch, Phys. Rev. Lett. 111, 185301 (2013).

[35] H. Miyake, G. A. Siviloglou, C. J. Kennedy, W. C. Burton, and W. Ketterle, Phys. Rev. Lett. 111, 185302 (2013).

[36] L.-J. Lang, X. Cai, and S. Chen, Phys. Rev. Lett. 108, 220401 (2012).

[37] P. Marra, R. Citro, and C. Ortix, Phys. Rev. B 91, 125411 (2015).

[38] F. Mei, S.-L. Zhu, Z.-M Zhang, C. H. Oh, and N. Goldman, Phys. Rev. A 85, 013638 (2012).

[39] S. Ganeshan, K. Sun, and S. Das Sarma, Phys. Rev. Lett. 110, 180403 (2013).

[40] H. Hu, C. Cheng, Z. Xu, H.-G. Luo, and S. Chen, Phys. Rev. B 90, 035150 (2014).

[41] F. Zhang, C. L. Kane, and E. J. Mele, Phys. Rev. Lett. 111, 056403 (2013).

[42] T. Fukui, Y. Hatsugai, and H. Suzuki, J. Phys. Soc. Jpn. 74, 1674 (2005).

[43] A. Altland and M. R. Zirnbauer, Phys. Rev. B 55, 1142 (1997).

[44] A. P. Schnyder, S. Ryu, A. Furusaki, and A. W. W. Ludwig, Phys. Rev. B 78, 195125 (2008).

[45] J.-S. Heron, C. Bera, T. Fournier, N. Mingo, and O. Bourgeois, Phys. Rev. B 82, 155458 (2010).

[46] Y. Sun, W. M. Choi, H. Jiang, Y. Y. Huang, and J. A. Rogers, Nat. Nanotechnol. 1, 201 (2006).

[47] T. S. Yarden and E. Joselevich, Nano Lett. 10, 4742 (2010).

[48] C. L. dos Santos and P. Piquini, Phys. Rev. B 81, 075408 (2010).

[49] D. Liang and X. P. A. Gao, Nano Lett. 12, 3263 (2012). 